\title{
Non classical Potter's sequence: a rare complication of chronic oligohydramnios
}

\author{
Shivali Bhalla, Sandesh Ganjoo, Prabhleen Kapoor, Varun Kaul, Amanpreet Sethi \\ Correspondence: Dr Shivali Bhalla, Senior Resident, Department of Obstetrics \& \\ Gynaecology, Guru Gobind Singh Medical College \& Hospital , Faridkot, Punjab, India. \\ Email - manasmansha@gmail.com
}

Distributed under Attribution-NonCommercial-ShareAlike 4.0 International (CC BY-NC-SA 4.0)

\begin{abstract}
Chronic oligohydramnios due to any cause can have grave foetal prognosis. One of the rare complications is Potter's sequence which occurs as a result of intrauterine mechanical compression of the foetus due to decreased liquor volume. This case report aims to give an insight into this rare complication. Classical Potter's sequence is due to renal cause whereas non classical, a still rarer variant of Potter's sequence is due to non renal cause. We describe a case of a lady at 38 weeks of gestational age with chronic oligohydramnios due to prolonged leaking per vaginum and severe intrauterine growth restriction. She delivered a congenitally malformed male baby with features of Potter's sequence. The renal abnormalities were conspicuously absent in our case, suggesting a diagnosis of non classical Potter sequence.
\end{abstract}

Keywords: Oligohydramnios sequence, VATER syndrome, Potter facies, limb hypoplasia.

Chronic oligohydramnios can lead to life threatening foetal complications like pulmonary hypoplasia, cord compression, intrauterine foetal compression leading to limb deformities, abdominal wall defects and Potter's sequence. Potter's syndrome (or Potter's sequence or Oligohydramnios sequence), is a rare complication of oligohydramnios. The term was coined by the pathologist Edith Potter, in 1946, to describe the facial characteristics of infants with bilateral renal agenesis and oligohydramnios. ${ }^{1}$ The term was initially used to refer to cases caused by bilateral renal aplasia (True Potter's sequence), however, nowadays, the term applies to atypical morphological appearance of the baby due to any underlying cause of oligohydramnios (Premature rupture of membranes, foetal growth restriction, post maturity or foetal chromosomal anomalies etc). The Potter's syndrome may be classified into various types the causes being renal and non - renal (Table 1). A retrospective analysis of children with Potter syndrome found that $21.25 \%$ had bilateral renal agenesis, $47.5 \%$ had cystic renal dysplasia, $25 \%$ had obstructive uropathy, and 5.25\% had other non -renal defects. ${ }^{2}$

The pathogenesis of Potter 's sequence is thought to be intrauterine compression of the growing fetus due to severe oligohydramnios leading to physical deformities, most common of which is "Potter's facies" (Figure 1). The latter is characterized by low set ears, receding chin, redunded fold of skin beneath the cheeks, flattened nasal bridge, parrot beak appearance of nose, prominent epicanthal fold. Other features of Potter's sequence

Received: $23^{\text {rd }}$ August 2018. Accepted: $16^{\text {th }}$ October 2018.

Bhalla S, Ganjoo S, Kapoor P, Kaul V, Sethi A. Non classical Potter's sequence: a rare complication of chronic oligohydramnios. The New Indian Journal of OBGYN. 2019; 5(2): 146-9 
Table 1: Classification of Potter's Syndrome

Classic Infant has bilateral renal agenesis (BRA), (malformation of the ureteric bud). True BRA also presents with bilateral agenesis of the ureters.

Type 1 Type I is due to autosomal recessive polycystic kidney disease (ARPKD)

Type 2 Complete agenesis or absence of one kidney and the remaining solitary kidney being small and malformed.

Type 3 Type III is due to Autosomal dominant polycystic kidney disease (ADPKD).

Type 4 Type IV occurs when a longstanding obstruction in either the kidney or ureter leads to cystic kidneys or hydronephrosis.

Non Another cause of Potter sequence (oligohydramnios or

classic anhydramnios) can be the rupturing of the foetal membranes.

\section{Case report}

We present the case of a 25 year old female, gravida 2 with history of one spontaneous abortion at $4^{\text {th }}$ month of gestation, referred to us from a peripheral health centre at 38 weeks of gestation. She presented with history of draining liquor per vaginum since $16^{\text {th }}$ week of pregnancy. Her doppler ultrasonography suggestive of severe intra-uterine growth restriction with foetal parameters corresponding to only 28 weeks of gestation, breech presentation, abnormal doppler velocimetry of umbilical arteries and severe foetal bradycardia (foetal heart

include: limb deformities (which include bowing of legs, clubbed feet, limb hypoplasia etc) ${ }^{3}$; pulmonary hypolpasia; ophthalmological malformations ${ }^{4}$ (cataract, prolapsed of lens, angiomatous malformation of optic disc area etc); cardio-vascular abnormalities ${ }^{5}$ ( ventricular septal defect, patent ductus arteriosus etc). Potter's sequence associated with bilateral renal agenesis is usually associated with a number of abnormalities like such as caudal dysgenesis, VATERL (Vertebral anomalies, Anal atresia, Cardiac defects, Tracheoesophageal fistula, Renal defects, Limb defects) ${ }^{6}$, caudal dysplasia syndrome, and isolated anomalies of the cardiovascular, skeletal, and central nervous systems ${ }^{7-9}$. These abnormalities can add to the morbidity and increased mortality in these patients. Males have an increased incidence of the Potter syndrome. Medical management of neonates diagnosed with Potter's sequence depends upon their renal function, respiratory status and associated congenital anomalies. In neonates with classical Potter sequence with bilateral renal agenesis, further treatment may not help and the prognosis is grave. However non classical Potter sequence due to rupture of membranes during gestation have a higher survival rate and demand proper assessment, resuscitation and management for better neonatal outcome .

We describe a case of a female with chronic oligohydramnios and severe intrauterine growth restriction who delivered a congenitally malformed baby along with features suggestive of Potter's syndrome. rate $90-100$ beats per $\mathrm{min}$ ) .On examination, her vitals were stable, pulse rate $70 / \mathrm{min}$, blood pressure $130 / 80 \mathrm{~mm}$ of $\mathrm{Hg}$. Her respiratory and cardiovascular examination did

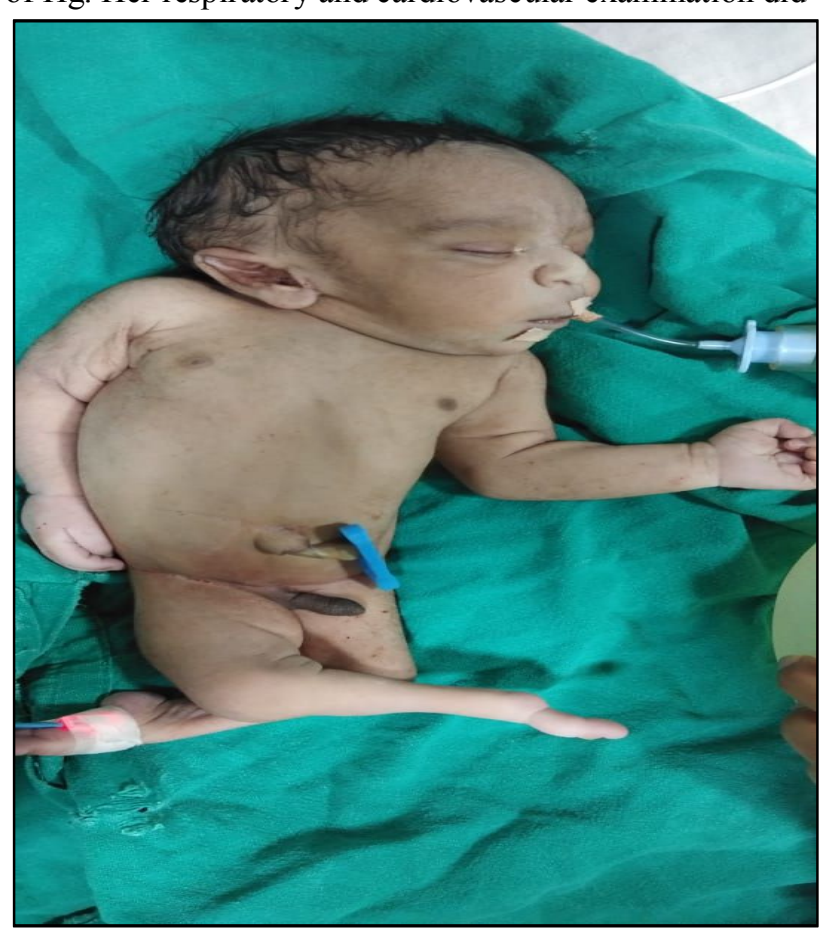

Figure 1: Neonate with Potter's sequence with characteristic physical deformities, "Potter 's facies" as characterized by low set ears, receding chin, redunded fold of skin beneath the cheeks, flattened nasal bridge and parrot beak appearance of nose. 
not reveal any abnormality. On per abdominal examination, fundal height corresponded to only 30 weeks of gestation, uterus was relaxed, liquor seemed almost absent and foetal heart rate was 90/min. On per vaginum examination os was $2 \mathrm{~cm}$, cervix early effaced, with breech presentation. Emergency doppler ultrasonography at our hospital showed a single live foetus with severe bradycardia with foetal heart rate of only 72 beats per minute in breech presentation with sonographic maturity of 29 weeks 6 days; with biparietal diameter $79.8 \mathrm{~mm}$, femur length $41.7 \mathrm{~mm}$ and abdominal circumference $248.4 \mathrm{~mm}$; liquor absent; severe utero placental insufficiency with reversal of forward diastolic flow in umbilical artery and abnormal middle cerebral artery doppler velocimetry. However no gross congenital malformation was detected.

The patient was immediately taken up for caesarean section to salvage the compromised baby. Asphyxiated male baby was delivered with 5 min APGAR score of 2 . The baby was immediately intubated and shifted to neonatal intensive care unit. The baby showed gross congenital malformations with characteristic Potter's facies with characteristic features like low set ears, receding chin, redunded fold of skin beneath the cheeks, flattened nasal bridge, parrot beak appearance of nose, prominent epicanthal fold. The baby had severe limb deformities with right lower limb hypoplasia. The right foot was undeveloped and popliteal fossa absent. Genitourinary malformations like absence of scrotum and absent anal opening was noted. The baby also had deformity of the lumbar spine. The baby had severe respiratory distress and had recurrent seizures. Baby could not survive and expired within 60 minutes of delivery. As the baby expired soon after delivery, no further investigations could be done for the baby. The relatives refused foetal autopsy. The mother had an otherwise uneventful post operative recovery and was discharged after stitch removal on $7^{\text {th }}$ post operative day.

\section{Discussion}

Potter's sequence may be due to renal or non renal cause. Classical Potter's sequence is said to occur in foetuses with bilateral renal agenesis leading to oligohydramnios. However, the broad term also includes the foetuses with features of Potter's sequence due to any underlying cause of oligohydramnios. Her antenatal ultrasonography showed normal foetal kidneys ruling out bilateral renal agenesis as the cause of Potter's sequence in this case. Hence our case seems to be a case of non classical Potter's sequence which is a very rare occurrence. Chest $\mathrm{X}$ ray of this baby showed pulmonary hypoplasia. X ray of lower extremities revealed absence of right foot, bowing of legs, absent popliteal fossa and fracture in left femur bone. Fracture femur in this case can be attributed to mechanical compression of the baby owing to long standing oligohydramnios.

However the baby had associated congenital malformations like spine deformities and absent anal opening, suggesting features of VATER abnormalities. It is to be noted here that VATER association with Potter's sequence point towards a diagnosis of bilateral renal agenesis as the underlying cause but renal abnormality was conspicuously absent in this baby. Relatives did not consent to foetal autopsy and karyotype, due to which we could not ascertain the details of the associated genetic or chromosomal abnormalities of the baby.

\section{Conclusion}

Potter's sequence is a rare but known complication of chronic oligohydramnios associated with grave foetal prognosis. Prevention and timely diagnosis of the underlying cause of oligohydramnios help to improve the neonatal outcome. Whereas classical Potter sequence demands termination of early pregnancy, owing to underlying defects like bilateral renal agenesis, the cases of non classical Potter's sequence can usually be prevented and treated to help provide better neonatal outcome.

\section{Conflict of interest: None. Disclaimer: Nil.}

\section{References}

1.Potter EL. Facial characteristics of infants with bilateral renal agenesis. American Journal of Obstetrics and Gynecology. 1946; 51: 885-8.

2.National Center for Advancing Translational Sciences. Translational Science of Rare Diseases. Maryland: NCATS; 2011

3.Fantel AG, Shepard TH. Potter syndrome. Nonrenal features induced by oligoamnios. Am J Dis Child. 1975; 129(11):1346-7. 
4.Ginsberg J, Buchino JJ, Menefee M, Ballard E, Husain I. Multiple congenital ocular anomalies with bilateral agenesis of the urinary tract. Ann Ophthalmol. 1979; 11(7):1021-9.

5.Greenwood RD, Rosenthal A, Nadas AS. Cardiovascular malformations associated with congenital anomalies of the urinary system. Observations in a series of 453 infants and children with urinary system malformations. Clin Pediatr (Phila). 1976; 15(12):1101-4.

6.Prouty LA, Myers TL. Oligohydramnios sequence (Potter's syndrome): case clustering in northeastern Tennessee. South Med J. 1987; 80(5): 585-92.

7.Kadhim HJ, Lammens M, Gosseye S, Gadisseux JF, Evrard P. Brain defects in infants with Potter syndrome (oligohydramnios sequence). Pediatr Pathol. 1993; 13(4): 519-36.

8.Preus M, Kaplan P, Kirkham TH. Renal anomalies and oligohydramnios in the cerebro-oculofacio-skeletal syndrome. Am J Dis Child. 1977; 131(1): 62-4.
9.Tonni G, Azzoni D, Ventura A, Ambrosetti F, De Felice C. Multicystic dysplastic kidney (Potter type II syndrome) and agenesis of corpus callosum (ACC) in two consecutive pregnancies: a possible teratogenic effect of electromagnetic exposure in utero. Fetal Pediatr Pathol. 2008; 27(6): 264-73.

\footnotetext{
Shivali Bhalla ${ }^{1}$, Sandesh Ganjoo ${ }^{2}$, Prabhleen Kapoor ${ }^{3}$, Varun Kaul ${ }^{4}$, Amanpreet Sethi ${ }^{5}$

${ }^{1}$ Senior Resident, Department of Obstetrics and Gynaecology; ${ }^{2}$ Assistant Professor, Department of Obstetrics and Gynaecology; ${ }^{3}$ Junior Resident, Department of Obstetrics and Gynaecology; ${ }^{4}$ Assistant Professor, Department of Pediatrics; ${ }^{5}$ Assistant Professor, Department of Pediatrics, Guru Gobind Singh Medical College \& Hospital , Faridkot, Punjab, India.
} 